\title{
Gamma-ray free-electron lasers: Quantum fluid model
}

\author{
H.M. Silva ${ }^{1}$, A. Serbeto ${ }^{2 *}$, R.M.O. Galvão ${ }^{3}$, J.T. Mendonça ${ }^{3}$, and L. F. Monteiro ${ }^{2}$ \\ ${ }^{1}$ Centro Brasileiro de Pesquisas Físicas, 22290-180, RJ, Brazil \\ ${ }^{2}$ Instituto de Física, Universidade Federal Fluminense, \\ Campus da Praia Vermelha, Niterói, RJ, 24210-346, Brazil and \\ ${ }^{3}$ Instituto de Física, Universidade de São Paulo, São Paulo, SP, 05508-090, Brazil
}

(Dated: July 4, 2021)

\begin{abstract}
A quantum fluid model is used to describe the interaction of a nondegenerate cold relativistic electron beam with an intense optical wiggler taking into account the beam space-charge potential and photon recoil effect. A nonlinear set of coupled equations is obtained and solved numerically. The numerical results show that in the limit of plasma wave-breaking, an ultra-high power radiation pulse is emitted at the $\gamma$-ray wavelength range, which can reach an output intensity near the Schwinger limit depending of the values of the FEL parameters, such as detuning and input signal initial phase at the entrance of the interaction region.
\end{abstract}

PACS numbers:

It is well known that the free-electron laser(FEL) conceived by Madey 1 is a source of coherent and tunable ligth with modulated frequency, which can generate very short wavelengths. In the standard configuration a magnetostatic wiggler is used to induce a transverse motion in the relativistic electron beam, such that the kinetic energy of the beam is released into electromagnetic stimulated radiation energy, which obeys the approximate resonance condition $\lambda_{s}=\lambda_{w} / 2 \gamma^{2}$, where $\lambda_{s}, \lambda_{w}$ and $\gamma$ are the stimulated radiation wavelength, wiggler period, and normalized relativistic beam energy, respectively. However, more recntly, it has been realized that an electromagnetic wiggler can alternatively be used to modulate the transverse beam motion. In this case, a counter-propagating laser pulse, working as a wiggler, changes the resonance condition to $\lambda_{s}=\lambda_{w} / 4 \gamma^{2}$, with $\lambda_{w}$ being the laser pulse wavelength. Today the FEL can produce coherent radiation in the X-ray range of operation [2, 3, which opens new possibilities of research of matter at atomic and molecular scales, with possible applications in fundamental and applied physics 4. The enhancement of the FEL emission to ultra-short wavelength down to the Gamma-ray range, will provide access to a wide range of experimental possibilities to study matters at sub-atomics scales.

In the microwave regime of operation, the FEL is described by the equations of motion of each single electron in the beam under the external electromagnetic field, and the wave equation of the electromagnetic field, with an external beam current. Assuming that all ponderomotive potential wells are identical in steady state, and neglecting beam slippage effects, the coupled system is analyzed within a unit ponderomotive potential well. Hence, electrons in the potential well are modelled by a discrete ensemble of initially uniform macro-particles. The nonlinear interaction between electrons and radiation field generates nonlinear electron bunching inside the potential well, exciting a collective wave emission mode. This is the basic mechanism of a FEL, where the emitted photon momentum recoil is not larger than the electron beam momentum spread. As the radiation frequency gets significantly higher, this standard description of particles in potential wells has to be reconsidered. In the X-ray regime, for instance, the longitudinal dimension of the ponderomotive potential well is so small that, for any reasonable beam density, not all the potential wells would be occupied, and there will be not find more than one electron found per well. However, the progress in the construction of FEL devices and the need to reach new levels of energy and ultra- short wavelengths of the laser radiation and the beam oscillations requires that the quantum characteristic of the FEL interaction be properly considered. Taking photon recoil into account, when the photon energies become either comparable or larger than the energy of the incoming relativistic beam, i.e, $\hbar \omega_{s} / \gamma_{e} m_{e} c^{2} \approx \hbar k_{s} / \gamma_{e} m_{e} c \geq 1$, the number of photons per electromagnetic mode becomes very small, thus justifying a quantum description. In this sense new interesting and different theoretical quantum models have been proposed [5-13. Here, we will follow the quantum fluid model pointed out in Refs. [11, 12] in order to describe the collective interaction of the electromagnetic waves and a nondegenerate cold relativistic electron beam. In this model, the electromagnetic waves are treated classically and the electron beam as a quantum cold plasma, which can be described as a quantum plasma fluid [14, 15, that incorporates the quantum recoil effect (or quantum diffraction) given by the Bohm potential term in the Euler-like equation of motion. In the steady-state regime, the quantum FEL dynamics in one-dimension is described by the following set of fluid equations 12 ]

\footnotetext{
* serberto@if.uff.br
} 


$$
\begin{gathered}
v \frac{\partial p}{\partial \xi}=-\frac{\partial}{\partial \xi}[V-e \phi]+\frac{\hbar^{2}}{2 m_{e} \gamma_{e}^{2}} \frac{\partial}{\partial \xi}\left[\frac{1}{\sqrt{n}} \frac{\partial^{2} \sqrt{n}}{\partial \xi^{2}}\right] \\
\frac{\partial n v}{\partial \xi}=0
\end{gathered}
$$

where, $\xi=\left(z-v_{e} t\right), m_{e}$ is the electron rest mass, $v_{e}$ is the unperturbed beam velocity, $\gamma_{e}=1 / \sqrt{1-v_{e}^{2} / c^{2}}=$ $\sqrt{1+p_{e}^{2} / m_{e}^{2} c^{2}}$ is the electron Lorentz factor, $V$ is the ponderomotive potential, and $\phi$ is the space-chage potential, which satisfys the Poisson equation, viz, $\partial^{2} \phi / \partial \xi^{2}=4 \pi e\left(n-n_{b}\right)$. Here, $n_{b}$ is the average beam density and $n$ is the density of the electron fluid element, which moves with velocity(momentum) $v(p)$. Now, let us consider that the electron beam interacts with a scattered radiation propagating in the positive $\hat{z}$ direction (the same as the electron beam) and with a constant amplitude optical wiggler, which propagates in the counter direction, given by $\vec{a}_{s}=1 / \sqrt{2}\left[\hat{e} a_{s}\left(z-v_{e} t\right) \exp \left(i k_{s} z-i \omega_{s} t\right)+c . c\right]$ and $\vec{a}_{w}=1 / \sqrt{2}\left[\hat{e} a_{w} \exp \left(-i k_{w} z-i \omega_{w} t\right)+c . c\right.$, respectively, where $\hat{e}=\hat{x}+i \hat{y}$ is the unitary polarization vector. Here, $a_{w}=e A_{w} / m_{e} c^{2}\left(a_{s}=e A_{s} / m_{e} c^{2}\right)$ is the normalized optical(radiation) wiggler amplitude with $k_{w}\left(k_{s}\right)$ and $\omega_{w}\left(\omega_{s}\right)$ being the wavenumber and frequency of the optical wiggler(radiation). Hence, the ponderomotive potential $V$ associated with these fields, which acts on the electron beam, can be defined as $V=\gamma m_{e} c^{2}$, where $\gamma=\sqrt{\gamma_{e}^{2}+\overrightarrow{a_{t}} \cdot \overrightarrow{a_{t}}}$ and $\overrightarrow{a_{t}}=\overrightarrow{a_{w}}+\overrightarrow{a_{s}}$ are the beam relativistic factor during the interaction and total normalized potential vector. Introducing these fields into the electromagnetic wave equation and considering that the transverse current, $\vec{J}_{\perp}=-e n \vec{v}_{\perp}$, for $\vec{v}_{\perp}=e\left(\vec{A}_{s}+\vec{A}_{w}\right) / \gamma m_{e} c$, due to the transverse momentum conservation, in the laboratory frame, and assuming that $a_{s}$ is a slowly varying envelope amplitude in space and time, the evolution equation for the scattered radiation, $a_{s}$, can be written as

$$
\left(\beta_{s}-\beta_{e}\right) \frac{\partial}{\partial \theta} a_{s}-i \bar{\delta} a_{s}-\frac{i}{2} \frac{\omega_{p}^{2}}{\gamma k_{l} c \omega_{s}}\left(1-\frac{n}{n_{b}}\right) a_{s}=-\frac{i}{2} \frac{\omega_{p}^{2}}{\gamma k_{l} c \omega_{s}} \frac{n}{n_{b}} a_{w} e^{-i \theta}
$$

where $\theta=k_{l} z-\omega_{l} t$ is the ponderomotive wave phase with $k_{l}=k_{s}+k_{\omega}$ and $\omega_{l}=\omega_{s}-\omega_{w}$. Notice that this phase can be rewritten as $\theta=k_{l}\left(z-v_{e} t\right)$ assuming that $v_{e} \approx v_{r}$, where $v_{r}=\omega_{l} / k_{l}$ is the electron resonant velocity. Here, $\beta_{s}=c k_{s} / w_{s}$ is the normalized radiation group velocity, $\omega_{p}^{2}=4 \pi e^{2} n_{b} / m_{e}$ is the squared plasma frequency, and $\bar{\delta}=\delta / c=\beta_{e}-\beta_{r}=\left(\gamma_{e}-\gamma_{r}\right) / \gamma_{r}^{3}$ is the detuning parameter [8].

Assuming that $v(\xi=0)=0, V(\xi=0)=\gamma_{0} m_{e} c^{2}, \gamma_{0}=\gamma(\xi=0), \phi(\xi=0)=0$, and $n(\xi=0)=n_{b}$, Eqs. $(1)$ and $(2)$ can be integrated to give, in a normalized form, the following nonlinear pendulum equation, viz.,

$$
\frac{\partial^{2} P}{\partial \theta^{2}}+2 \sigma^{2}\left[\gamma_{0}-\gamma+\Phi\right] P=0
$$

with the space-charge potencial, $\Phi=e \phi / m_{e} c^{2}$, which obeys the normalized Poisson equation, given by

$$
\frac{\partial^{2} \Phi}{\partial \theta^{2}}=\Omega_{p}^{2}\left(P^{2}-1\right)
$$

where $P=\sqrt{n / n_{b}}, \Omega_{p}^{2}=\omega_{p}^{2} / \omega_{s}^{2}, \gamma=\left(\gamma_{e}^{2}+a_{w}^{2} / 2+\left|a_{s}\right|^{2} / 2+a_{s}^{*} a_{w} e^{-i \theta}+c . c\right)^{1 / 2}$, and $\sigma=m_{e} c \gamma_{e}^{3 / 2} / \hbar k_{l}$. It should be notice that this equation is formally identical to the equation obtained in Ref. [16] for a quantum plasma in rest, $\gamma_{e}=1$, in the laboratory frame, and in the absence of an optical wiggler, which gives a relativistic solitary wave in a cold plasma in the classical limit $(\hbar \rightarrow 0)$. Considering that $\left(\beta_{s}-\beta_{e}\right) \approx 1 / 2 \gamma_{e}^{2}$ and redefining $a_{s}=a e^{-i \theta}$, Eq. $(3)$ can be rewritten as

$$
\frac{\partial a}{\partial \theta}-i\left[1+2 \gamma_{e}^{2} \bar{\delta}+\Omega_{0}^{2}\left(1-P^{2}\right)\right] a=-i \Omega_{0}^{2} a_{w} P^{2}
$$

where $\Omega_{0}^{2}=\gamma_{e} \omega_{p}^{2} / \omega_{s}^{2}$ and $\gamma \approx \gamma_{e}$ has been used in the denominators of Eq.(3). Using the polar representation for the scattered radiation amplitude, $a$, in such a way that $a \rightarrow \operatorname{aexp}(i \varphi)$, we obtain the following set of real equations, i.e,

$$
\begin{gathered}
\frac{\partial a}{\partial \theta}=-\Omega_{0}^{2} a_{w} P^{2} \sin \varphi \\
\frac{\partial \varphi}{\partial \theta}=1+2 \gamma_{e}^{2} \bar{\delta}+\Omega_{0}^{2}\left(1-P^{2}\right)-\Omega_{0}^{2} \frac{a_{w} P^{2}}{a} \cos \varphi .
\end{gathered}
$$


Equations (4) - (8) form a set of nonlinear coupled basic equations that describes a quantum FEL pumped by an optical wiggler, where the relativistic electron beam is treated as a cold quantum plasma. These equations are solved numerically considering an electron beam with normalized energy, $\gamma_{e}=60$, and density, $n_{b}=1.0 \times 10^{9} \mathrm{~cm}^{-3}$, propagating in the positive $\hat{z}$ direction, interacting with counter-propagating laser wiggler whose the amplitude, $a_{w} \approx 0.6$, which is equivalent to a laser intensity $I_{w} \approx 2.3 \times 10^{18} \mathrm{~W} / \mathrm{cm}^{2}$ for a wavelenght $\lambda_{w}=6.50 \times 10^{-5} \mathrm{~cm}(650 \mathrm{~nm})$. It should be noticed that such intensity is compatible with existing laser tecnology. In order to enhance the stimulated radiation, a signal, with initial amplitude $a(\theta=0)=1.0 \times 10^{-5}$, propagating in the same direction as the beam, is introduced. Due to the FEL resonance condition, $k_{s} \approx 4 k_{w} \gamma_{e}^{2}$, in the interaction region, a very short wavelenght, $\lambda_{s} \approx 4.52 \times 10^{-9} \mathrm{~cm}$, is obtained, corresponding to an input signal with intensity of $I_{s} \approx 1.32 \times 10^{17} \mathrm{~W} / \mathrm{cm}^{2}$. It should be noticed that the quantum effect correction on the resonance condition, for low energy electron beam, has been neglected[12].

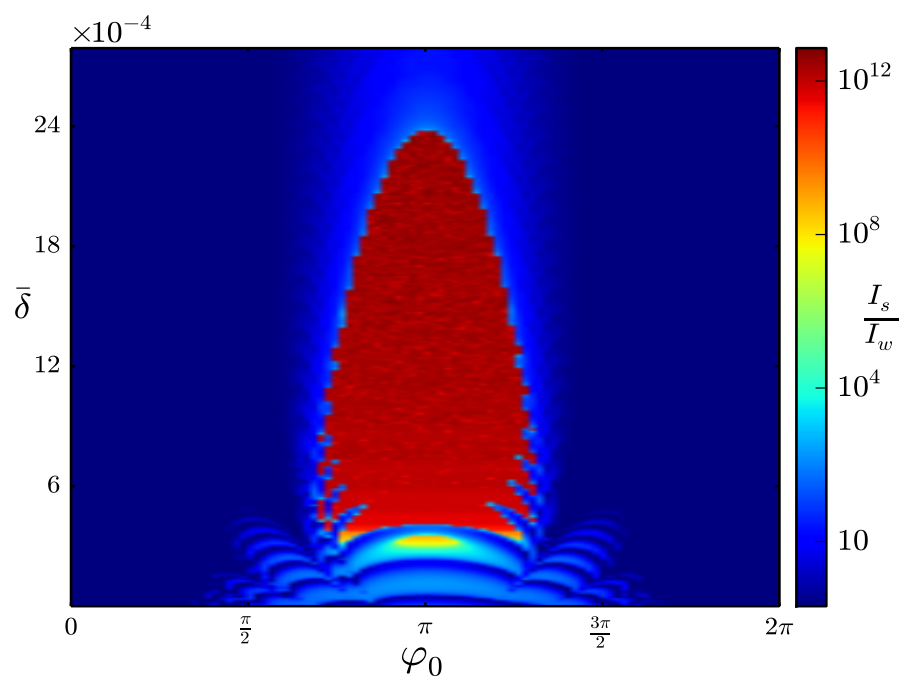

FIG. 1: Output radiation intensity for differents values of the detuning, $\bar{\delta}$, and initial phase, $\varphi_{0}$, of the input signal at the entrance of the interaction region.

Fig.1 displays the output laser intensities excited during the FEL interaction, for different values of the detuning and initial phase at the entrance of the interaction region. We can see that, depending of the values of the detuning and initial phase, the system can reach output intensity towards the Schwinger limit $\left(10^{29} \mathrm{~W} / \mathrm{cm}^{2}\right)$, which corresponds to the critical electric field where electron-positron pairs can be created(orange to red colours shown in the figure). Of course, our model fails for values of output intensities above this critical limit, where the FEL interaction should be treated from the point of view of quantum electrodynamics. This high intensity FEL emission occurs due to strong nonlinear space-charge wave profiles, excited by the relativistic ponderomotive potential, $V$. This produces density perturbations that lead to profile wave steepening, with the formation of intense electron beam density maxima, leading to space-chage wave-breaking regime, where the maximum energy transfer to radiation field is observed. See Fig.2 for an illustration. Clearly, such a behavior depend strongly of the initial phase of the radiation signal, for a range of values of the detuning parameter. Naturally, the main purpose in this regime is to obtain high intensity FEL emission. Indeed, it follows from the numerical rusults shown in Fig.1 that intense output power near de Schwinger limit is possible in narrow bands of the input parameters (light red). Outside of these bands the scattered radiation intensity drops markedly (dark blue), whereas in other regions (light blue, green and yellow) moderate output intensities can be observed $\left(10^{20}-10^{26} \mathrm{~W} / \mathrm{cm}^{2}\right)$, depending of the values of the detuning and initial phase parameters. As the detuning increases, for instance, $\bar{\delta} \approx 2.5 \times 10^{-3}$, and keeping the same initial phase as $\varphi_{0}=\pi$, the output radiation intensity decreases drastically, as shown in Fig.3.

We have used a quantum fluid model to describe the $\gamma$ - Free-electron laser interaction, using a classical ponderomotive potential, to drive the interaction of a nondegenerate cold relativistic electron beam pumped by an optical wiggler, taking into account the space-charge potential effect which plays a fundamental role on the stability of the system. The relevance of the detuning and initial phase parameters on the FEL dynamics, in order to obtain high intensity output radiation is described. The FEL interaction is analyzed in the parameter space, showing that a strong nonlinear space-charge wave can eventually be generated, with intense energy transfer to the scattered ra- 


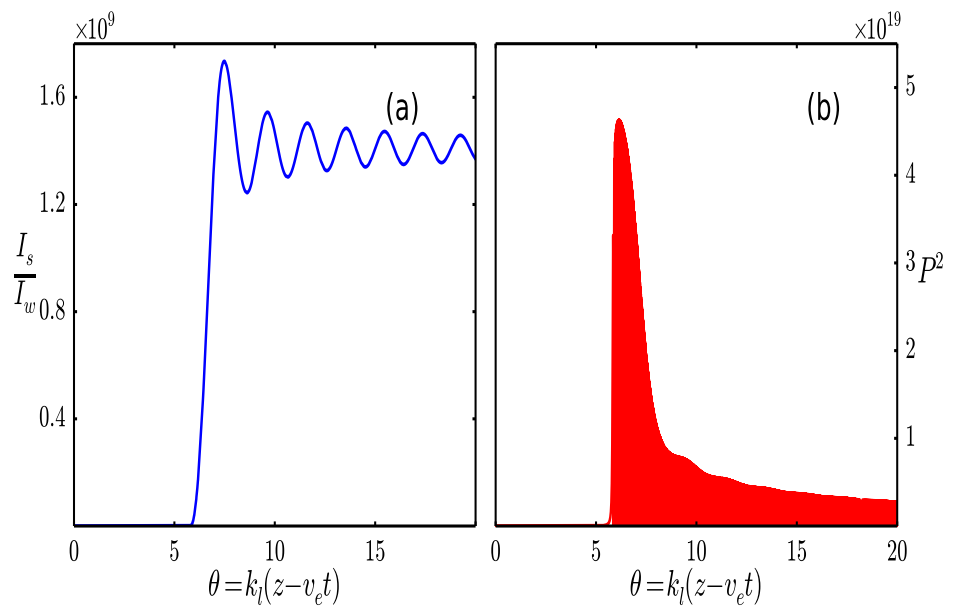

FIG. 2: Evolution of output intensity(a) and electron density profile(b) for detuning, $\bar{\delta} \approx 3.47 \times 10^{-4}$, and initial phase, $\varphi_{0}=\pi$.

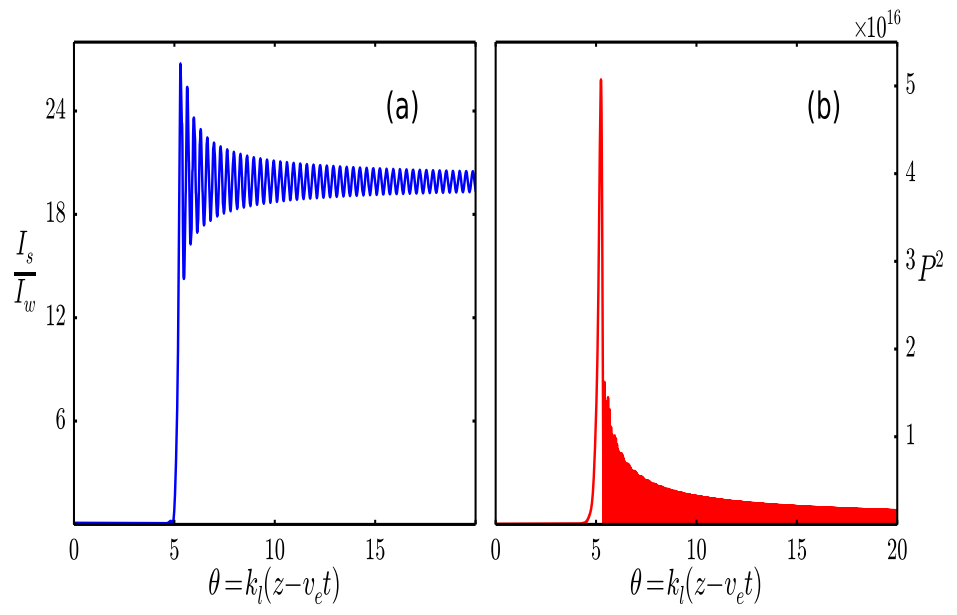

FIG. 3: Evolution of output intensity(a) and electron density profile(b) for detuning, $\bar{\delta}=2.5 \times 10^{-3}$, and initial phase, $\varphi_{0}=\pi$.

diation spectrum, up to the Schwinger critical limit, in a way very similar to electromagnetic wave scattered by a relativistic mirror [17]. A more completed quantum description of this process could be used, following the approach developed in our recent publication[18. Finally, it should be pointed out that intrinsic relativistic quantum effects, such as those associate with spin and with electron-positron coupling, were ignored. We should also say that the present quantum fluid model brakes down for an output power beyond the Schwinger limit, which is the domain of quantum electrodynamic(QED).

This work has been supported by the Brazilian Agency CNPq and by the State of São Paulo Agency FAPESP. One of the authors (HTS) would like to thank to Prof. Rubem Luis Sommer of the Brazilian Center for Physical Research (CBFP) for the support to use the computer cluster and to Diego Gonzales Chavez for his great help during the long time computation. 
[1] J.M.J. Madey, J. Appl. Phys., 42, 1906 (1971).

[2] E. Hand, Nature, 461, 708 (2009).

[3] T. Ishikawa et al., Nature Photonic, 6, 540 (2012).

[4] T. Tajima, New Trends in Plasma Physics, 29,(2009)

[5] J. K. Mclever and M. V. Fedorov, Sov. Phys. JETP, 49(6), 1012(1979).

[6] I. V. Smetanin, Laser Physics, 7(2), 318(1997)

[7] G. Preparata, Phys. Rev. A, 38, 233 (1988).

[8] R. Bonifacio, N. Piovella, and G. R. M. Robb, Nucl. Instrum. Methods A, 543, 645(2005).

[9] R. Bonifacio, M. M. Cola, N. Piovella, and G. R. M. Robb, Europhys.Lett., 69, 55(2005).

[10] A. Serbeto, J. T. Mendonça, K. H. Tsui, and R. Bonifacio, Phys. Plasmas, 15, 013110(2008).

[11] A. Serbeto, L. F. Monteiro, K. H. Tsui K. H., and J. T. Mendonça, Plasma Phys. Control. Fusion, 51, 124024(2009).

[12] L. F. Monteiro, A. Serbeto, K.H. Tsui, J. T. Mendonça, and R. M. O. Galvão, Phys. Plasmas, 20, 073101(2013).

[13] B. Eliasson and P. K. Shukla, Phys. Rev. E,85,(2012).

[14] F. Haas, Theory of Quantum Plasmas (Springer, New York, 2011).

[15] J. T. Mendonça, Phys. Plasmas, 18, 062101 (2011).

[16] P. K. Shukla and B. Eliasson, Phys. Rev. Lett., 51, 096401(2007).

[17] Sergei V. Bulanov, Timur Esirkepov and Toshiki Tajima, Phys. Rev. Lett., 91(8), 085001(2003).

[18] J. T. Mendonça, A. Serbeto, and R. M. O Galvão, Phys. Plasmas, 21, 053109(2014). 